University of Rhode Island

DigitalCommons@URI

Mechanical, Industrial \& Systems Engineering

Faculty Publications

Mechanical, Industrial \& Systems Engineering

2018

\title{
A minimal mechanics model for mechanosensing of substrate rigidity gradient in durotaxis
}

\author{
Bahador Marzban \\ University of Rhode Island
}

Xin Yi

Hongyan Yuan

University of Rhode Island, yuanhy3@sustech.edu.cn

Follow this and additional works at: https://digitalcommons.uri.edu/mcise_facpubs

The University of Rhode Island Faculty have made this article openly available.

Please let us know how Open Access to this research benefits you.

This is a pre-publication author manuscript of the final, published article.

Terms of Use

This article is made available under the terms and conditions applicable towards Open Access Policy Articles, as set forth in our Terms of Use.

Citation/Publisher Attribution

Marzban, B., Yi, X. \& Yuan, H. Biomech Model Mechanobiol (2018) 17: 915. https://doi.org/10.1007/

s10237-018-1001-3

Available at: https://doi.org/10.1007/s10237-018-1001-3

This Article is brought to you for free and open access by the Mechanical, Industrial \& Systems Engineering at DigitalCommons@URI. It has been accepted for inclusion in Mechanical, Industrial \& Systems Engineering Faculty Publications by an authorized administrator of DigitalCommons@URI. For more information, please contact digitalcommons-group@uri.edu. 


\title{
A minimal mechanics model for mechanosensing of substrate rigidity gradient in durotaxis
}

\author{
Bahador Marzban ${ }^{1}$, Xin $\mathrm{Yi}^{2}$, Hongyan Yuan ${ }^{1 *}$ \\ ${ }^{1}$ Department of Mechanical, Industrial \& Systems Engineering, University of Rhode \\ Island, Kingston, RI 02881 USA \\ ${ }^{2}$ Department of Mechanics and Engineering Science, College of Engineering, Peking \\ University, Beijing 100871, China
}

*Corresponding Author: hongyan_yuan@uri.edu

\begin{abstract}
Durotaxis refers to the phenomenon in which cells can sense the spatial gradient of the substrate rigidity in the process of cell migration. A conceptual two-part theory consisting of the focal adhesion force generation and mechanotransduction has been proposed previously by Lo et al. to explain the mechanism underlying durotaxis. In the present work, we are concerned with the first part of the theory: how exactly is the larger focal-adhesion force generated in the part of the cell adhering to the stiffer region of the substrate? using a simple elasticity model and by assuming the cell adheres to the substrate continuously underneath the whole cell body, we show that the mechanics principle of static equilibrium alone is sufficient to account for the generation of the larger traction stress on the stiffer region of the substrate. We believe that our model presents a simple mechanistic understanding of mechanosensing of substrate stiffness gradient at the cellular scale, which can be incorporated in more sophisticated mechanobiochemical models to address complex problems in mechanobiology and bioengineering.
\end{abstract}

\section{Introduction}

It has been shown that biological cells can sense and respond to a variety of mechanical cues of their microenvironment, such as matrix rigidity (Lo et al. 2000), matrix topology (Uttayarat et al. 2005), matrix dimensionality (Harunaga and Yamada 2011), shear flow (Li et al. 2002), interstitial flow (Polacheck et al. 2014), cell-cell and cell-matrix adhesions (Chen et al. 2004), and cell shape constraints (Parker et al. 2002). These mechanical stimuli play a critical regulatory role in many biological functions such as cell proliferation (Nelson et al. 2005), cell motility (Lo et al. 2000; Parker et al. 2002), and differentiation (Engler et al. 2006). Understanding the mechanisms underlying mechanosensing has become the focus of intensive experimental and theoretical studies (Vogel and Sheetz 2006; Gao et al. 2011; Yuan and Gao 2012; Borau et al. 2014). In the present study, we are interested in durotaxis, a termed coined by Lo et al. (Lo et al. 2000), which refers to the substrate rigidity-guided cell migration. They showed that (see Fig. 1a) when a fibroblast cell crawled from the stiffer side (i.e., the darker region) of the substrate toward the softer side (i.e., the brighter region), the cell made a 90-degree turn at the interface. 
a

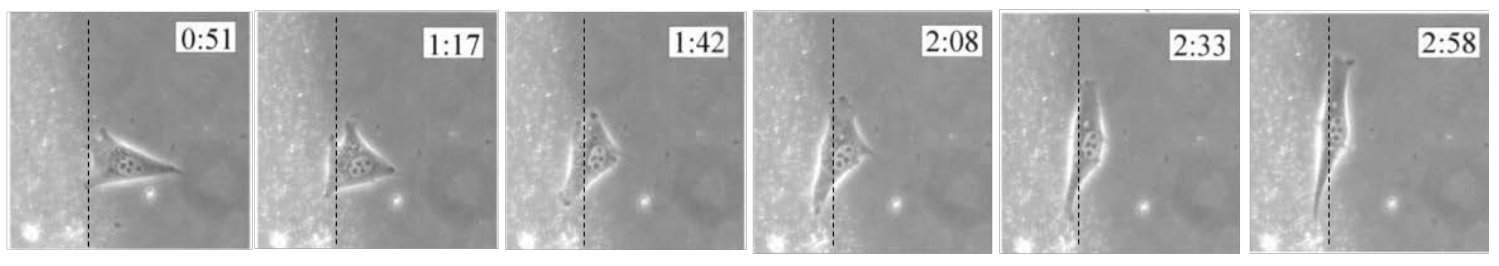

b

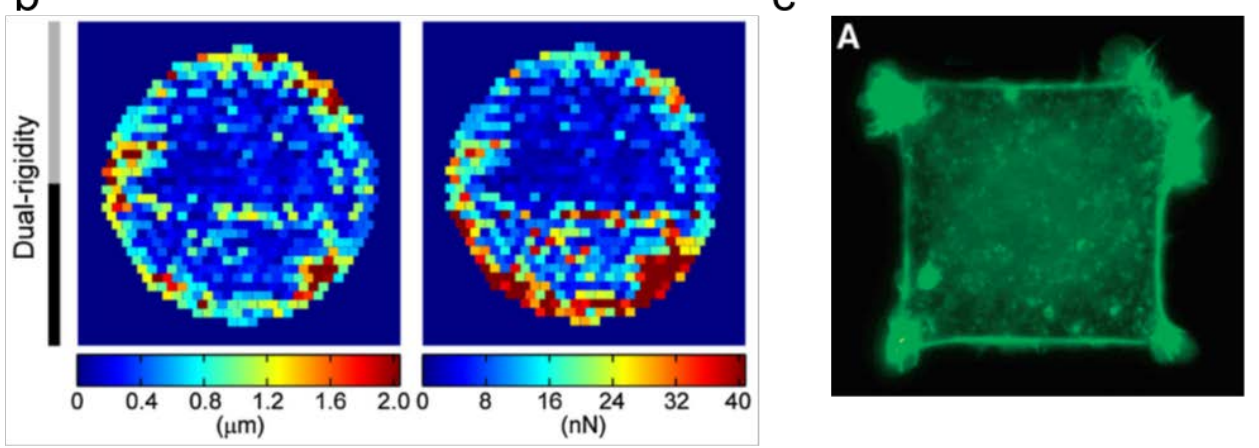

Figure 1. Previous experimental observations on single-cell mechanosensing. (a) The phenomenon of durotaxis (reprinted with permission from (Lo et al. 2000)): a cell crawls from the stiffer side of the substrate toward the softer side and turned $90^{\circ}$ at the interface (the dotted line is an approximation of the rigid-to-soft interface). (b) Traction stress under a circular cell crossing step-rigidity boundary (reprinted with permission from (Breckenridge et al. 2014)) (c) Lamellipodia extension in a square cell (reprinted with permission from (Brock et al. 2003)).

Because the importance of durotaxis in physiology and pathology, the molecular and subcellular mechanisms underlying mechanotransduction has attracted considerable attention (Wang et al. 2001; Mitrossilis et al. 2009; Plotnikov et al. 2012; Wong et al. 2014). Biomechanics models where single cells and cell-substrate linkages were modeled as elastic springs or elasticity theory, have been developed to account for rigidity sensing (Moreo et al. 2008; Borau et al. 2011, 2014). These models were only applied to the scenario where the individual cell was treated either as a point mass (Moreo et al. 2008) or an small volume element (Borau et al. 2011). In a series of studies (Zemel et al. 2010a, b; Cohen and Safran 2016), by modeling the individual cells or stress fibers as force dipoles distributed in continuum elastic 2D or 3D substrates, the researchers developed biomechanics models to interpretate mechanosensing mechanisms and to study the effect of mechanosensing on cell shape, stress fiber orientation, and synchronized beating of cardiomyocytes. On the other hand, the effect of durotaxis on cell migration dynamics on the long time have also been studied. For example, in the cell migration model by (Yu et al. 2017), substrate rigidity-dependence is taken into account by assuming focal adhesions are correlated with substrate stiffness. In the single cell migration model by (Allena et al. 2016), substrate rigidity-dependence is considered by assuming differential cell-substrate adhesions strengh on substrates with different rigidity. Thus, in these durotaxis models, substrate-rigidity-dependence is used as the assumption in the cell migration models. To the best of our knowledge, in previous models, the spatial distribution of the substrate 
rigidity within the single cell domain is constant. The substrate rigidity is either changed for the whole cell or changed only when the cell moves from one location to another (during migration). The main difference between these models and our model is that we examine how a single cell senses the local substrate rigidity difference within the single cell domain. Therefore, our model can provide a more direct interpretation on how the cell sense the rigidity gradient.

In their original paper (Lo et al. 2000), Lo et al. proposed a two-part theory for the detection of the spatial gradient of the substrate rigidity as follows. In the first part of their theory, the cytoskeleton-focal adhesion-substrate linkages are considered as elastic springs (with spring constant $k$ ); for the same amount of elastic energy input $U$ (from the active actomyosin contraction) to pull these springs, the spring force $F$ generated is larger at the stiffer region of the substrate underneath the cell (Because $U=\frac{F^{2}}{2 k} \rightarrow F=\sqrt{2 k U}$, thus for the same $U$, larger $k$ results in larger $F$ ). In the second part the theory, the stronger force leads to a higher level of activation of force-sensitive proteins through conformational changes, which in turn leads to migration-related cellular responses such as upregulation of lamellipodia extension. The second part of the theory is referred to as mechanotransduction (Chen 2008) in the literature. This two-part theory is directly supported by other experimental observations. For example, in a work by Breckenridge et al. (Breckenridge et al. 2014), traction stress under a circular cell crossing step-rigidity boundary were measured using elastomeric micropost arrays. They found that the traction stress is higher on the stiffer half of the circular island (see Fig. 1b), which supports the first part of the theory. In another work (Brock et al. 2003), the authors found lamellipodia grow preferentially from the corners of square cells (see Fig. 1c). The corners of convex polygonal shapes are known to be the spots where high traction stress is generated when the cell contracts (Yuan et al. 2017). Together these findings support the second part of the durotaxis thoery that larger focal adhesion force leads to more lamellipodia extensions.

In this work, we are concerned with the first part of the theory: how exactly is the larger focal adhesion force generated in the part of the cell adhering to the stiffer region of the substrate? The assumption of Lo et al. that the same energy is provided for pulling is not without pitfalls, since it is not straightforward as how the generation of the same mechanical energy is ensured at the different sub-regions of the cell by the cell's active contractile apparatus. Another (and easier) approach to calculate the force is to consider the static equilibrium of the cell. The migrating speed of fibroblast cells is very slow ( 1 $\mu \mathrm{m} / \mathrm{min}$ ), considering the stress fibers are in a state of isometric tension, thus the cell at any time instant can be considered to be in a quasi-static equilibrium. Therefore, the static equilibrium holds for the whole mechanical system composed of the cell and the elastic substrate.

Using the method of static equilibrium, a simple generic model based on active matter theory has been devised by Marcq et al. (Marcq et al. 2011), in which the cytoskeleton was modeled as two parallel elements (one passive spring and one active contractile element), and the 1D cell connects to the substrate springs only at the two ends (see Fig. 2a). Their 
model is sufficient to explain the experimental findings (Ghibaudo et al. 2008; Mitrossilis et al. 2009) where the magnitude of the traction stress increases with the substrate rigidity. However, it cannot explain the rigidity gradient sensing (i.e., different traction stress at the two ends of the same cell): the static equilibrium implies that the adhesion forces at the two ends of the cell should be the same, regardless of disparate substrate-spring stiffness. This is the paradox that was raised in a review by (Danuser et al. 2013).

The assumption that the 1D cell only adheres to the substrate at the two ends oversimplifies the problem. In fact, by dropping this assumption, the abovementioned paradox can be resolved. Considering that the cell adheres to the substrate in the whole cell domain, in the present study, we show that the static equilibrium of the cell is sufficient to yield the rigidity gradient-dependent traction force distribution. The remainder of the paper is organized as follows. We first present the simple elasticity model for 1D and 2D cell adhering to an elastic substrate. For the 1D cell, we will derive analytical solutions and present results from the parametric studies. For the 2D cell, we will use the finite element method (FEM) to numerically solve the equilibrium equations. We then compare the modeling results with the three experimental observations listed in Fig. 1.

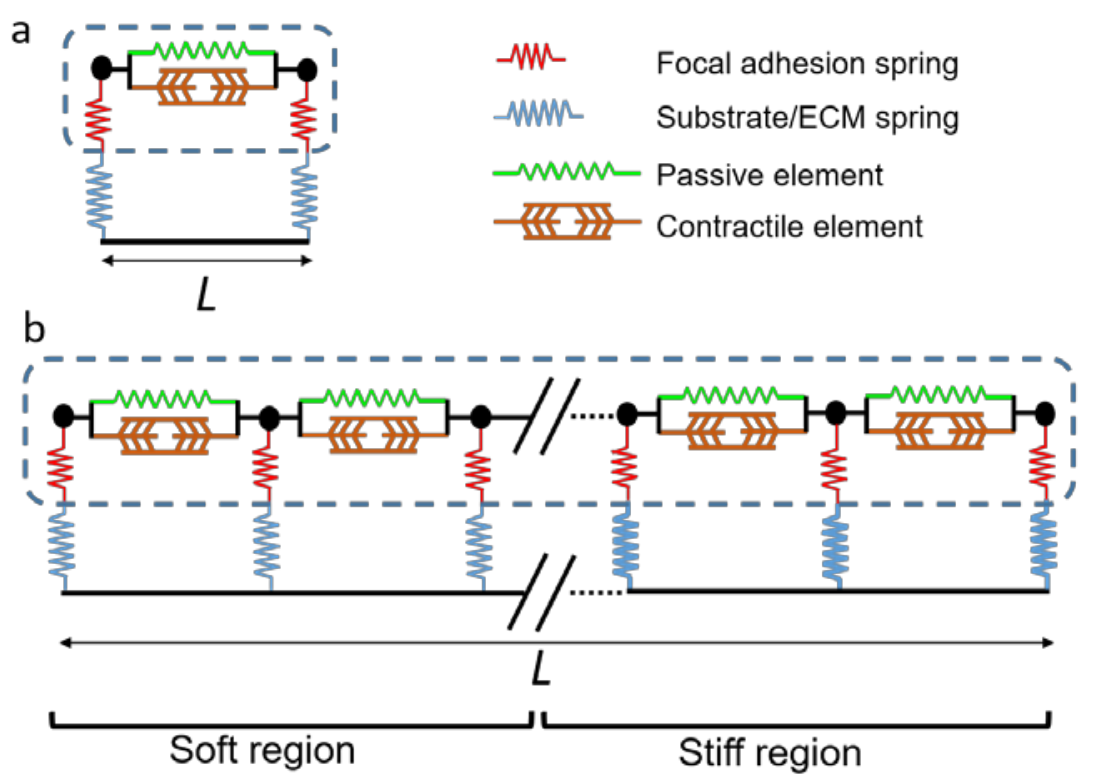

Figure 2. (a) Schematic representation of a single-cell model by Marcq et al. (Marcq et al. 2011), where the cell adheres to the substrate only at the two ends. (b) Schematic representation of our single-cell model where the cell adheres to the substrate in the whole cell domain. The left half of the cell ahdere to a soft region, while the right half of the cell adheres to a stiff region of the substrate. The cytoskeleton is composed of a passive spring and an active contractile element. Note that the FA and substrate springs, alghouth drawn in a vertical direction, resist displacement in the horizontal direction in the 1D and 2D model.

\section{Model description}




\section{D Model}

We first present a 1D model of a cell adhering to an elastic substrate. As shown in Fig. 2b, the cytoskeleton of the cell is modelled a 1D strip of length $L$, adhering to the substrate through the focal adhesions. The focal adhesions and the substrate are treated as linear springs of stiffness $k_{F A}$ and $k_{E C M}$, respectively. Note that $k_{F A}$ and $k_{E C M}$ denotes the stiffness of continuum springs so they are in units of stress per unit length, instead of force per unit length. Because the substrate is modeled as isolated springs (i.e., elastic interaction within the substrate is neglected), the substrate considered in our model can be thought as the elastomeric micropost arrays (Breckenridge et al. 2014), rather than a conitnouum elastic substrate. The active actomyosin contraction shortens the 1D cell and the shortening is resisted by the passive compoment of the cytoskeleton and the substrate (see the schematic in Fig. 2b).

Constitutive relations of the cytoskeleton have been previously studied intensively. Timedependent constitutive relations based on Hill's law of muscle contraction have been devised previously to capture the dynamic process of actomyosin contraction, such as for stress fibers (Deshpande et al. 2006; Mitrossilis et al. 2009; Borau et al. 2014) or for myofibrils (McCain et al. 2014; Aratyn-Schaus et al. 2016). In this work, for simplicity, the final state of the dynamic models when contraction stress reaches isometric tension and strain rate becomes zero is considered, which yields a time-independent consituttive relation for the $1 \mathrm{D}$ cytoskeleton:

$$
\sigma_{x}=E \varepsilon_{x}+\sigma_{c}
$$

where $\sigma_{x}$ is the overall cytoskeleton stress, $E$ is the Young's modulus of the passive component of the cytoskeleton, $\sigma_{c}$ is the isometric tension due to the active actomyosin contraction, $\varepsilon_{x}=\frac{\partial u_{x}}{\partial x}$ is the strain, $u_{x}$ is the displacement along the axis of the $1 \mathrm{D}$ cell (i.e., $x$-axis).

The static equilibirum equation of the $1 \mathrm{D}$ cell is

$$
h \frac{\partial \sigma_{x}}{\partial x}-T_{x}=0
$$

where $h$ is the thickness of the cell and is assumed to be a constant for simplicity, $T_{x}$ is the traction stress exerted on the substrate by the cell. Because the focal adhesion is connected to the substrate spring in series, $T_{x}$ is also the stress experienced by the focal adhesion. Therefore, we here use the phrases "focal adhesion stress" and "traction stress" interchangeably in this paper. Traction stress $T_{x}$ can be calculated as

$$
T_{x}=k_{c s} u_{x}
$$

where $k_{c s}$ is the equivalent spring constant of the cell-substrate linkage composed of the focal adhesion spring and the substrate spring, as illustrated in Fig. 2b. Because the two springs are in series, we have

$$
k_{c S}=\left(k_{F A} k_{E C M}\right) /\left(k_{F A}+k_{E C M}\right)
$$

To model the rigidity gradient, we define step changes in substrate rigidity by

$$
k_{E C M}=\left\{\begin{array}{c}
k_{s}, x<0 \\
\alpha k_{s}, x>0
\end{array}\right.
$$


where $\alpha$ defines the ratio of rigidities of the two regions, which can be regarded as the gradient strength. Without loss of generality, the left half (i.e, $x<0$ ) is considered to be softer than the right half (i.e., $x>0$ ). Therefore, $\alpha \geq 1$ is imposed in our parametric studies. The stress-free boundary condition applies at the two ends: $\sigma_{x}(x= \pm L / 2)=0$, where $L$ is the length of the 1D cell. The stress continuity condition at the interface between the stiff and soft regions is $\sigma_{x}\left(x=0^{-}\right)=\sigma_{x}\left(x=0^{+}\right)$. Equations (1)-(5), along with the boundary and interface conditions, can be solved analytically for the displacement $u_{x}$, stress $\sigma_{x}$, and traction stress $T_{x}$.

\section{D Model}

To apply the model to cells cultured on 2D surface of elastic substrate, we extend the 1D model to the 2D. For the 2D model, Eq. (1)-(3) become

$$
\begin{gathered}
\sigma_{i j}=2 G e_{i j}+K \varepsilon_{k k} \delta_{i j}+\sigma_{c} \delta_{i j} \\
h \sigma_{i j, j}-T_{i}=0 \\
T_{i}=k_{c s} u_{i}
\end{gathered}
$$

where the indicial notation is used, summation over repeated indices is adopted, $\sigma_{i j}, e_{i j}$, and $\varepsilon_{i j}$ are stress tensor, deviatoric strain tensor, strain tensor, respectively, $G$ and $K$ are shear and bulk moduli of the cytoskeleton. the substrate rigidity gradient is modeled by defining $k_{E C M}$ as a function of Cartesian coordinates $(x, y)$. The finite element method (FEM) (Zienkiewicz et al. 2005) is used to numerically solve the differential equations of the 2D model, where 3-node triangle element is used for the spatial discretization.

\section{Results}

\section{D cell, when $\alpha=1$}

When the gradient strength parameter $\alpha=1$, meaning uniform rigidity underneath the cell, the 1D model can be readily solved for the displacement $u_{x}$ and traction $T_{x}$ as follows. Using the strain-displacement and constitutive relations, Eq. (2) can be rewritten as an linear second-order differential equation: $h E u_{x, x x}-k_{c s} u_{x}=0$. By defining $\beta=$ $\sqrt{\frac{k_{c s}}{E h}}$, the solution can be written as, $u_{x}=a e^{\beta x}+b e^{-\beta x}$. Imposing the stress-free boundary condition at two ends (i.e., $\sigma_{x}(x= \pm L / 2)=0$ ) and, the two unknown coefficients $a$ and $b$ are determined as: $a=-b=-\frac{\sigma_{c}}{E \beta} \frac{\gamma}{\gamma^{2}+1}$, where $\gamma=e^{-\frac{\beta L}{2}}$. Traction stress can be found using Eq. (3) as a function of $x$ as: $T_{\mathrm{x}}(x)=-k_{c s} \frac{\sigma_{c}}{E \beta} \frac{\gamma}{1+\gamma^{2}}\left(e^{\beta x}+\right.$ $e^{-\beta x}$ ). The magnitude of $T_{\mathrm{x}}(x)$ maximizes at the two ends of the cell (i.e., $x= \pm L / 2$ ). Denoting the magnitude of traction stress located at the two ends of the cell by $T_{\mathrm{END}}$, we have

$$
T_{\mathrm{END}}=k_{c s} \frac{\sigma_{c}}{E \beta} \frac{1-\gamma^{2}}{1+\gamma^{2}}
$$

where $k_{c S}$ is given in Eq. (4). Figure 3a plots $T_{\mathrm{END}}$ as a function of $k_{E C M}$, which shows that the traction stress reaches a plateau when $k_{E C M} \rightarrow \infty$, which implies there is a saturation value of traction stress or force at large substrate rigidity. This result has been previously shown in experiments and models (Ghibaudo et al. 2008; Mitrossilis et al. 2009; Marcq et 
al. 2011) Mathematically, this is simply because $k_{c s} \rightarrow k_{F A}$ when $k_{E C M} \rightarrow \infty$. Mechanically, this is because two springs in series is softer than any of the two springs. Therefore, when the substrate becomes rigid, the spring stiffness of the cell-substrate linkage becomes equal to the focal adhesion spring.

\section{D cell, when $\alpha>1$}

When $\alpha>1$, a step change of rigidity is present underneath the single cell (the left half is always softer than the right half). The analytical solution can be derived similar to the case of $\alpha=1$. Figure 3b and 3c show the solutions of traction stress $T_{x}$ and cytoskeletal stress $\sigma_{x}$, respectively. For the traction stress, positive sign means rightward pulling and negative sign means leftward pulling. Clearly, the traction stress magnitude is maximal at the cell edge on the stiff region (i.e., at the position $x / L=1 / 2$ ). With continuous adhesion to the substrate in the whole cell domain, traction stress is redistributed so that on the stiffer side the traction stress is within a shorter range but higher magnitude on average. On average, the higher cytoskeleton stress $\sigma_{x}$ is generated in the stiff region compared to the soft region. Note that traction stress is discontinuous at the interface (i.e., $x=0$ ). This is simply because the substrate rigidity is assumed to be discontinuous at the interface in our model (see Eq. 5, there is a step change of rigidity across $x=0$ ). If we assumed a linearvarying rigidity gradient, the traction stress would be continuous.

a

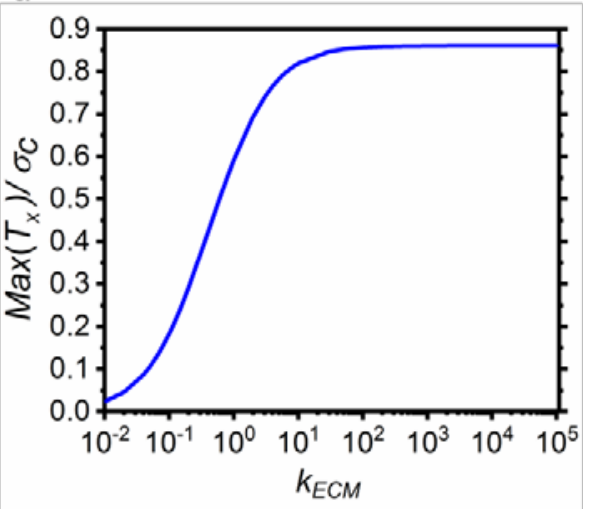

b

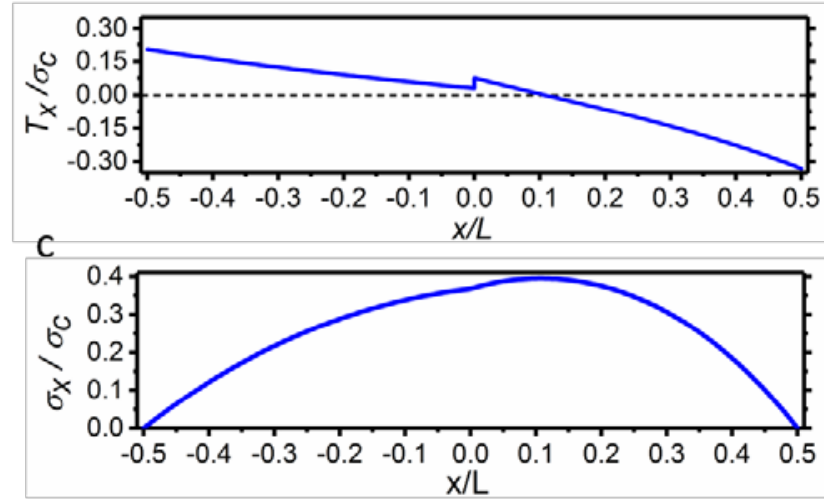

Figure 3. (a) Traction stress (scaled by $\sigma_{c}$ ) reaches a plateau at large substrate rigidity (in units of $\mathrm{kPa} / \mu \mathrm{m}$ ). (b) Analytical solution of traction stress $T_{x}$. (c) Analytical solution of cytoskeletal stress $\sigma_{x}$. Parameter values used: $L=20 \mu \mathrm{m}, k_{F A}=1 \mathrm{kPa} / \mu \mathrm{m}, k_{s}=0.1$ $\mathrm{kPa} / \mu \mathrm{m}, h=3 \mu \mathrm{m}, \sigma_{c}=4 \mathrm{kPa}, \alpha=3$, and $E=4 \mathrm{kPa}$. These parameter values are used for the remainder of the paper unless specifically mentioned.

These results imply that the static equilibrium alone can account for dependence of the traction stress on the rigidity gradient of the substrate, which is the first part of the rigiditygradient sensing theory by Lo et al. mentioned previously. With the onset of different forces in focal adhesion and cytoskeleton, the positive feedbacks between the traction stress and focal adhesion maturation and between the cytoskeleton tension and the stress fiber formation can further amplify the differences of these forces, and eventually result into disparate cellular responses through mechanotransduction pathways. 
Parametric studies were conducted to ascertain the sensitivity of the modeling results to the parameter values. We define the difference between the traction stresses at the left and right ends of the $1 \mathrm{D}$ cell as $\Delta T_{x}=\left|T_{x}(L / 2)\right|-\left|T_{x}(-L / 2)\right|$, where | | denotes the absolute value. Quantity $\Delta T_{x}$ represents the difference between the traction stresses on the soft and stiff regions of the substrate. Figure 4 plots the $\Delta T_{x}$ as a function of $\alpha$ for different values of $E$ and $k_{s}$. In both Fig. $4 \mathrm{a}$ and $4 \mathrm{~b}$, we see that the traction stress difference increases with $\alpha$, which implies that the gradient strength play an important role in durotaxis (Isenberg et al. 2009; Vincent et al. 2013). In Fig. 4a, we can see that $\Delta T_{x}$ increases as $E$ decreases, meaning softer the passive component of the cytoskeleton, larger difference of traction stress is produced. When the passive component of the cytoskeleton becomes stiffer, less contractile force is transmitted to the focal adhesion and consequently weaker dependence of focal adhesion stress on the substrate rigidity. In Fig. 4b, we can see that the ratio between the relative difference of traction stress between the soft and stiff regions (i.e., $\Delta T_{x} / T_{\max }$, where $T_{\max }=T_{x}(L / 2)$ ) increases with decreasing substrate rigidity $k_{s}$, which implies that if the mechanotransduction process detects the relative difference of traction stress, then softer substrate promotes durotaxis.
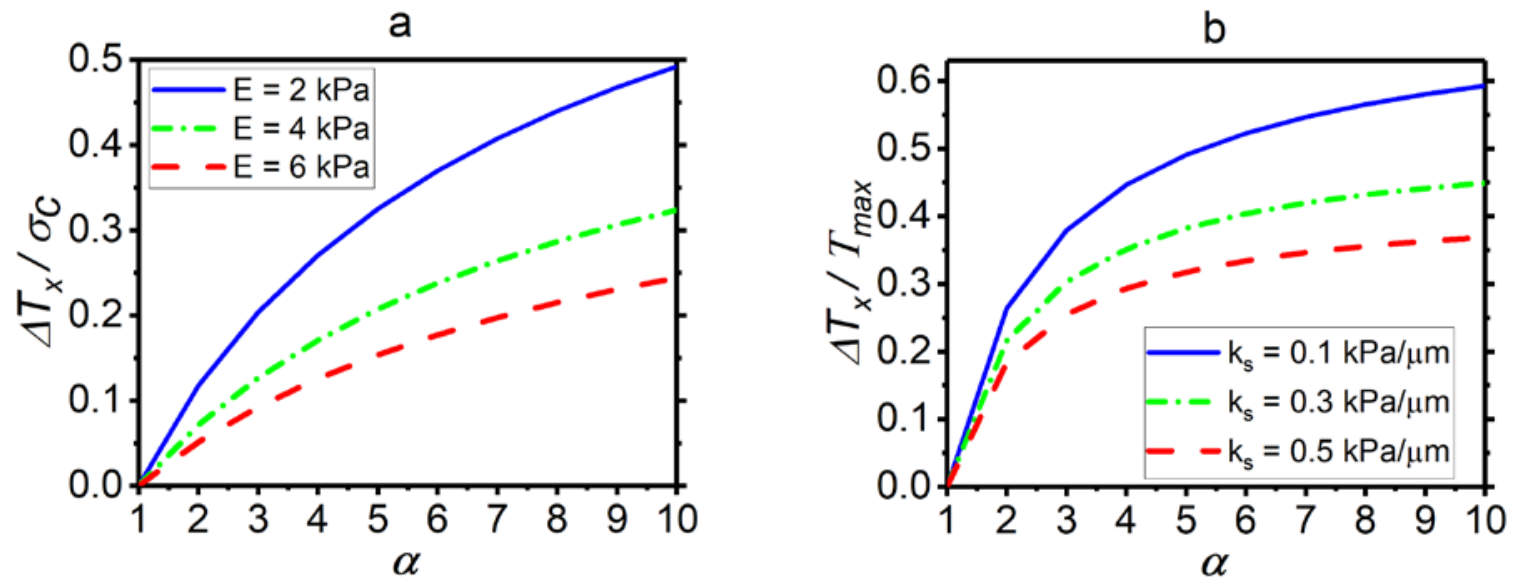

Figure 4. (a) Traction stress difference $\Delta T_{x}$ as a function of $\alpha$ for different values of $E$ when $k_{s}=0.1 \mathrm{kPa} / \mu \mathrm{m}$. (b) $\Delta T_{x} / T_{\max }$ as a function of $\alpha$ for different values of $k_{s}$ when $E=2 \mathrm{kPa}$.

\section{Computational results for $2 \mathrm{D}$ cells}

First, we show in Fig. 5a the FEM simulation results from the 2D cell model for a circular cell crossing a step-rigidity boundary (i.e., the upper half of the cell adheres to soft micropost arrays, the lower half of the cell adheres to stiff micropost arrays). As shown in Fig. 5a, the traction stress is higher on the perimeter of the cell, and it is higher on the lower half (stiff substrate) compared to the upper half (soft substrate). The displacement is slightly higher on the upper half than the lower half. Our modeling results in Fig. 5a are in good agreement with in the experimental results (Breckenridge et al. 2014) shown in Fig. $1 \mathrm{~b}$ (if we neglect the random noise in the experiment). Therefore, both the experiment and our model show that the cytoskeleton contraction in the single cell generates higher traction stress on the stiffer region of the substrate underneath the cell. 
Second, we show in Fig. 5b the model prediction of traction stress for the square cell. The traction stress concentrates to the edge of the square cell, and maximizes at the corners. This modeling result is correlated with the experimental data by (Parker et al. 2002; Brock et al. 2003) shown in Fig. 1c where the lamellipodia extensions were localized to the corners of square-shaped cells. This correlation supports the durotaxis theory proposed by (Lo et al. 2000): larger focal adhesion forces at the corners of the square cell are converted into protrusion signals via molecular mechanisms of mechanotransduction, which eventually lead to stronger lamellipodia extension. In the case of durotaxis, higher traction stresses are in the rigid side of the substrate and essentially the protrusion signals will be amplified in the rigid side rather than in the soft side.

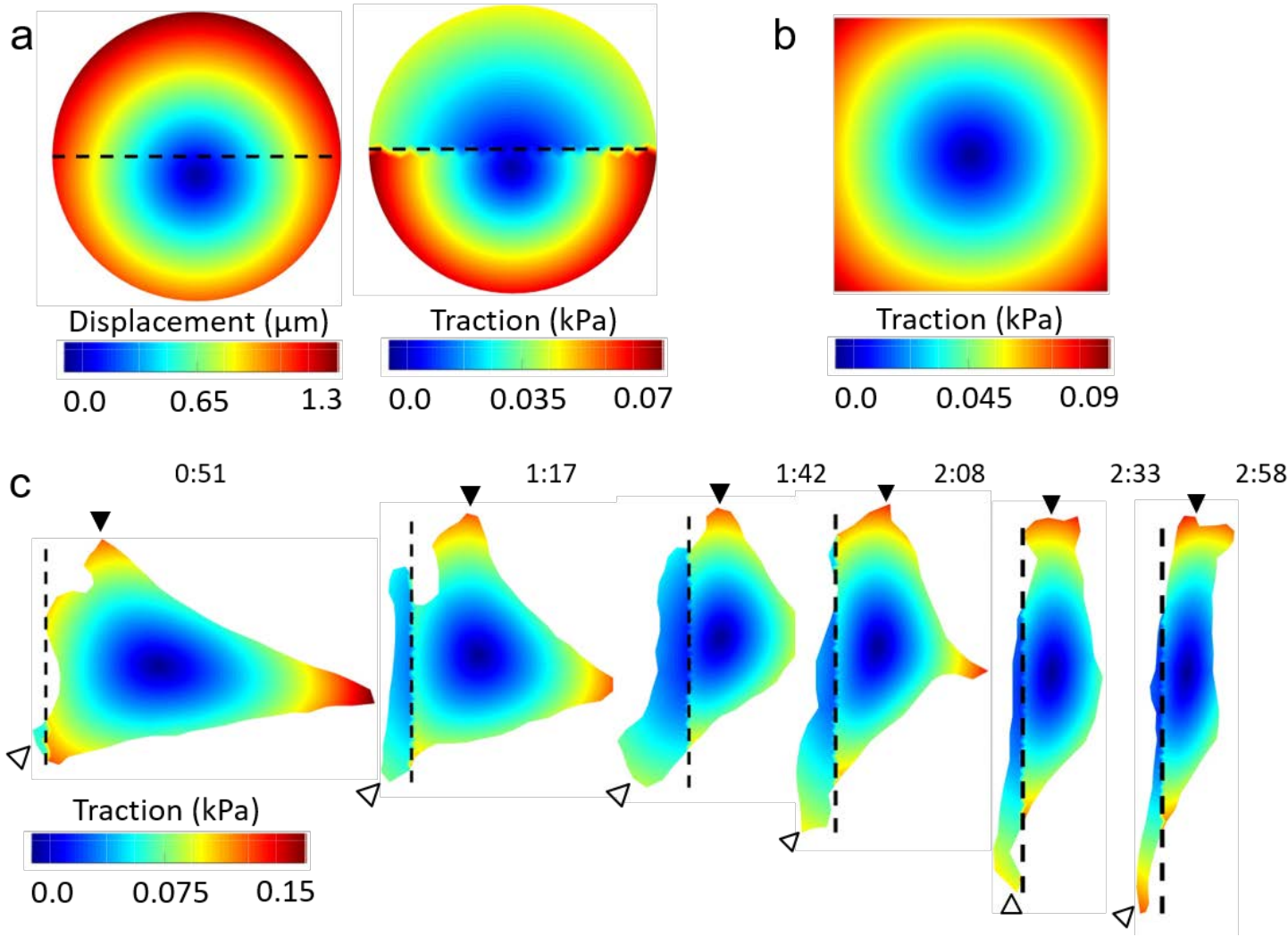

Figure 5. Finite element model predictions for 2D cells. (a) Displacement and traction stress of a circular cell crossing a step-rigidity boundary. (b) Traction stress for the square cell. (c) Traction stress distributions of the cell shown in Fig. 1a at the sequential time instants. (Parameter values used: $G=2.3 \mathrm{kPa}, K=5 \mathrm{kPa}$, cell area is $500 \mu \mathrm{m}^{2}$, other parameters are the same as $1 \mathrm{D}$ analytical model given in Fig. 3)

We then apply the 2D model to Lo et al.'s experiment (Fig. 1a) to calculate the traction stress distribution. A vertical line is picked (approximately based on the brightness change in the image) in Fig. 1a to be the interface between the soft and stiff sides of the substrate. Figure $5 c$ shows the traction stress distributions corresponding to the experimental images of Fig. 1a at the different time instants. One can see that the traction stress for the lamellipodia on the rigid side (solid arrowhead) is larger than that of the lamellipodia on the soft side (hollow arrowhead). If the larger force is converted into more protrusion signal, 
the lamellipodia on the right side (solid arrowhead) will become the dominant one, which eventually leads to the turning of the cell at the step-rigidity boundary. Note that the highest traction stress spot at the tail of the cell at the beginning (see Fig. 5c) does not result in a leading head is probably because the memory (in the molecular constitutes) of the head-totail polarization (Prentice-Mott et al. 2016), i.e., the new head will most likely to form near the original head.

\section{Conclusions}

In this work, we use a simple elasticity mechanics model to predict the traction stress (i.e., focal adhesion stress) for single adherent cells on the elastic substrate with rigidity gradient. The model predicts larger traction stress (i.e., larger focal adhesion stress because the traction stress is equal to the force experienced by the focal adhesion) on stiffer region of the substrate underneath a single cell. This minimal mechanics model provides a plausible answer for the first part of the durotaxis theory proposed by Lo et al. (Lo et al. 2000): how exactly is the larger focal adhesion force generated in the part of the cell adhering to the stiffer region of the substrate? We found that the principle of static equilibrium alone provides a mechanistic explanation to this question. We think our model has resolved the paradox that was raised in a review by (Danuser et al. 2013), which states that a static model cannot explain the rigidity sensing of a cell. Our model can be incorporated in more sophisticated mechanobiochemical models to address complex problems in mechanobiology and bioengineering.

\section{Acknowledgments}

H.Y. acknowledge funding support from the ASME Haythornthwaite Research Initiation Grant Award. X.Y. acknowledges the Thousand Youth Talent Plan Funds of China.

\section{Compliance with ethical standards}

\section{Conflicts of interest}

The authors declare that they do not have any conflict of interest.

\section{References}

Allena R, Scianna M, Preziosi L (2016) A Cellular Potts Model of single cell migration in presence of durotaxis. Math Biosci 275:57-70 . doi: 10.1016/j.mbs.2016.02.011

Aratyn-Schaus Y, Pasqualini FS, Yuan H, et al (2016) Coupling primary and stem cellderived cardiomyocytes in an in vitro model of cardiac cell therapy. J Cell Biol 212:389-97 . doi: 10.1083/jcb.201508026

Borau C, Kamm RD, García-Aznar JM (2011) Mechano-sensing and cell migration: a 3D model approach. Phys Biol 8:66008 . doi: 10.1088/1478-3975/8/6/066008

Borau C, Kamm RD, García-Aznar JM (2014) A time-dependent phenomenological model for cell mechano-sensing. Biomech Model Mechanobiol 13:451-462 . doi: 10.1007/s10237-013-0508-X

Breckenridge MT, Desai RA, Yang MT, et al (2014) Substrates with engineered step changes in rigidity induce traction force polarity and durotaxis. Cell Mol Bioeng 7:26-34 . doi: 10.1007/s12195-013-0307-6 
Brock A, Chang E, Ho CC, et al (2003) Geometric determinants of directional cell motility revealed using microcontact printing. Langmuir 19:1611-1617 . doi: 10.1021/la026394k

Chen CS (2008) Mechanotransduction - a field pulling together? J Cell Sci 121:32853292 . doi: $10.1242 /$ jcs.023507

Chen CS, Tan J, Tien J (2004) MECHANOTRANSDUCTION AT CELL-MATRIX AND CELL-CELL CONTACTS. Annu Rev Biomed Eng 6:275-302 . doi: doi:10.1146/annurev.bioeng.6.040803.140040

Cohen O, Safran SA (2016) Elastic interactions synchronize beating in cardiomyocytes. Soft Matter 12:6088-6095 . doi: 10.1039/C6SM00351F

Danuser G, Allard J, Mogilner A (2013) Mathematical modeling of eukaryotic cell migration: insights beyond experiments. Annu Rev Cell Dev Biol 29:501-528 . doi: 10.1146/annurev-cellbio-101512-122308

Deshpande VS, McMeeking RM, Evans AG (2006) A bio-chemo-mechanical model for cell contractility. Proc Natl Acad Sci U S A 103:14015-14020 . doi: 10.1073/pnas.0605837103

Engler AJ, Sen S, Sweeney HL, Discher DE (2006) Matrix elasticity directs stem cell lineage specification. Cell 126:677-689 . doi: 10.1016/j.cell.2006.06.044

Gao H, Qian J, Chen B (2011) Probing mechanical principles of focal contacts in cellmatrix adhesion with a coupled stochastic-elastic modelling framework. J R Soc Interface

Ghibaudo M, Saez A, Trichet L, et al (2008) Traction forces and rigidity sensing regulate cell functions. Soft Matter 4:1836-1843 . doi: 10.1039/b804103b

Harunaga JS, Yamada KM (2011) Cell-matrix adhesions in 3D. Matrix Biol 30:363-8 . doi: 10.1016/j.matbio.2011.06.001

Isenberg BC, Dimilla PA, Walker M, et al (2009) Vascular smooth muscle cell durotaxis depends on substrate stiffness gradient strength. Biophys J 97:1313-1322 . doi: 10.1016/j.bpj.2009.06.021

Li S, Butler P, Wang Y, et al (2002) The role of the dynamics of focal adhesion kinase in the mechanotaxis of endothelial cells. Proc Natl Acad Sci U S A 99:3546-3551 . doi: 10.1073/pnas.052018099

Lo CM, Wang HB, Dembo M, Wang Y-L (2000) Cell movement is guided by the rigidity of the substrate. Biophys J 79:144-152

Marcq P, Yoshinaga N, Prost J (2011) Rigidity sensing explained by active matter theory. Biophys J 101:26-29 . doi: 10.1016/j.bpj.2011.08.023

McCain ML, Yuan H, Pasqualini FS, et al (2014) Matrix elasticity regulates the optimal cardiac myocyte shape for contractility. Am J Physiol Heart Circ Physiol 306:H1525-39 . doi: 10.1152/ajpheart.00799.2013

Mitrossilis D, Fouchard J, Guiroy A, et al (2009) Single-cell response to stiffness exhibits muscle-like behavior. Proc Natl Acad Sci 106:18243-18248 . doi: 10.1073/pnas.0903994106

Moreo P, García-Aznar JM, Doblaré M (2008) Modeling mechanosensing and its effect on the migration and proliferation of adherent cells. Acta Biomater 4:613-621 . doi: 10.1016/j.actbio.2007.10.014

Nelson CM, Jean RP, Tan JL, et al (2005) Emergent patterns of growth controlled by multicellular form and mechanics. Proc Natl Acad Sci U S A 102:11594-11599 
Parker KK, Brock AL, Brangwynne C, et al (2002) Directional control of lamellipodia extension by constraining cell shape and orienting cell tractional forces. Faseb J 16:1195-1204

Plotnikov S V., Pasapera AM, Sabass B, Waterman CM (2012) Force fluctuations within focal adhesions mediate ECM-rigidity sensing to guide directed cell migration. Cell 151:1513-1527 . doi: 10.1016/j.cell.2012.11.034

Polacheck WJ, German AE, Mammoto A, et al (2014) Mechanotransduction of fluid stresses governs 3D cell migration. Proc Natl Acad Sci U S A 111:2447-52 . doi: 10.1073/pnas.1316848111

Prentice-Mott H V., Meroz Y, Carlson A, et al (2016) Directional memory arises from long-lived cytoskeletal asymmetries in polarized chemotactic cells. Proc Natl Acad Sci 113:201513289 . doi: 10.1073/pnas.1513289113

Uttayarat P, Toworfe GK, Dietrich F, et al (2005) Topographic guidance of endothelial cells on silicone surfaces with micro- to nanogrooves: Orientation of actin filaments and focal adhesions. J Biomed Mater Res Part A 75A:668-680 . doi: 10.1002/jbm.a.30478

Vincent LG, Choi YS, Alonso-Latorre B, et al (2013) Mesenchymal stem cell durotaxis depends on substrate stiffness gradient strength. Biotechnol J 8:472-484 . doi: 10.1002/biot.201200205

Vogel V, Sheetz M (2006) Local force and geometry sensing regulate cell functions. Nat Rev Mol Cell Biol 7:265-275

Wang HB, Dembo M, Hanks SK, Wang Y (2001) Focal adhesion kinase is involved in mechanosensing during fibroblast migration. Proc Natl Acad Sci U S A 98:11295300 . doi: 10.1073/pnas.201201198

Wong S, Guo W-H, Wang Y-L (2014) Fibroblasts probe substrate rigidity with filopodia extensions before occupying an area. Proc Natl Acad Sci 111:17176-17181 . doi: 10.1073/pnas.1412285111

Yu G, Feng J, Man H, Levine H (2017) Phenomenological modeling of durotaxis. Phys Rev E 96:1-6 . doi: 10.1103/PhysRevE.96.010402

Yuan H, Gao H (2012) On the mechanics of integrin clustering during cell-substrate adhesion. Acta Mech Solida Sin 25:467-472 . doi: 10.1016/S0894-9166(12)60041$\mathrm{X}$

Yuan H, Marzban B, Parker KK (2017) Myofibrils in Cardiomyocytes Tend to Assemble Along the Maximal Principle Stress Directions. J Biomech Eng 139: . doi: 10.1115/1.4037795

Zemel A, Rehfeldt F, Brown AEX, et al (2010a) Optimal matrix rigidity for stress-fibre polarization in stem cells. Nat Phys 6:468-473 . doi: 10.1038/nphys1613

Zemel A, Rehfeldt F, Brown AEX, et al (2010b) Cell shape, spreading symmetry, and the polarization of stress-fibers in cells. J Phys Condens Matter 22:194110 . doi: 10.1088/0953-8984/22/19/194110

Zienkiewicz OC, Taylor RL, Zhu JZ (2005) The Finite Element Method: its Basis and Fundamentals 